\title{
Transient Contrast Induced Encephalopathy After Carotid Artery Stenting; A Fact Not Fiction
}

\author{
Walid Hassan*, Mariam Hassan and Sahar Sharaf Eldin \\ Department of Cardiovascular Disease, International Medical Center Jeddah, Saudi Arabia
}

*Corresponding author: Walid Hassan, Department of Cardiovascular Disease, International Medical Center Jeddah, Saudi Arabia.

Received Date: November 23, 2018

Published Date: November 29, 2018

\section{Background}

This report is to emphasize the true existence of the rare phenomena of transient neurotoxic effect (encephalopathy) of the iodinated contrast after carotid angiography and stenting.

We describe an interesting case of a 91-year-old man with severe symptomatic left carotid subtotal stenosis with multiple comorbidities who developed a transient contrast induced encephalopathy shortly after carotid stenting and completely resolved within 24 hours.

\section{Case Report}

A 91-year-old male with history of multiple illness including; hypertension, diabetes, dyslipidemia, mild renal impairment, coronary artery disease and coronary bypass grafting 20 years ago. He presented to us with recurrent right sided transit ischemic attacks (TIAs), he is right-handed, fully oriented with no speech, sensory or motor deficit at the time of evaluation. His carotid arterial Doppler ultrasound showed critical left internal carotid artery stenosis $>90 \%$ with high peak systolic velocity (Figure 1), so he was referred for carotid angiogram and possible intervention as he is a high risk for carotid endarterectomy. Selective carotid/ cerebral angiography showed mild right carotid disease 30\% and identified a critical 90\% left internal carotid artery stenosis (Figure

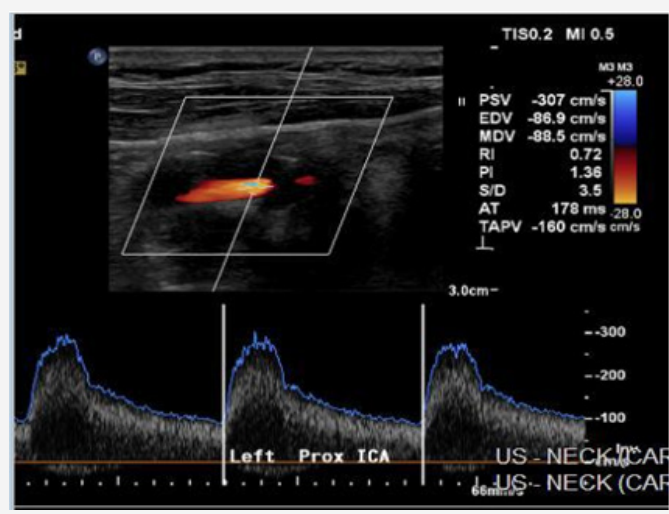

Figure 1: Carotid arterial Doppler ultrasound showed left internal carotid artery stenosis $>90 \%$ with high peak systolic velocity.
2). He underwent successful carotid stenting using Ambushed NAV 6 distal protection filter and $8 \times 6 \times 40 \mathrm{~mm}$ XACT self-expandable stent with excellent result (Figure 3), he received unfractionated heparin throughout the procedure with therapeutic activated clotting time (ACT), already on clopidogrel and received a total of $60 \mathrm{~mL}$ of picomolar, nonionic iodixanol (Viviparus) contrast. He was hemodynamically and neurologically intact, interactive and fully communicating during the procedure. Shortly (30 minutes) after the procedure he became lethargic and confused but no sensory or motor deficit, so we immediately repeated the cerebral angiography which revealed a wide patent stent with excellent flow to middle and anterior cerebral arteries as well as patent all distal branches (Figure 4, 5). Noncontract computed tomography showed no acute event as well as brain magnetic resonance imaging MRI (unremarkable diffusion, sulci and no cortical edema or acute defect) no brain alteration on diffusion-weighted magnetic resonance imaging (DW-MRI) (Figure 6). The patient was observed in the critical cardiac unit with frequent neurological check and was given intravenous fluid $1 \mathrm{~mL} / \mathrm{kg} /$ hour normal saline; he made a remarkable improvement after 24 hours and completely recovered back to his full cognition. He was seen two weeks and one month after the procedure, he was well, repeat carotid Duplex revealed patent carotid arteries, he continued to lead an active life.

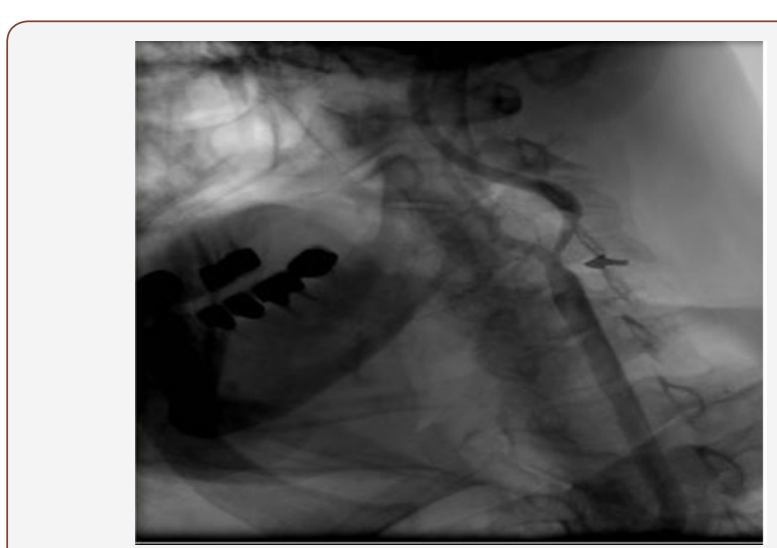

Figure 2: Selective carotid/cerebral angiography showed $90 \%$ left internal carotid artery stenosis. 


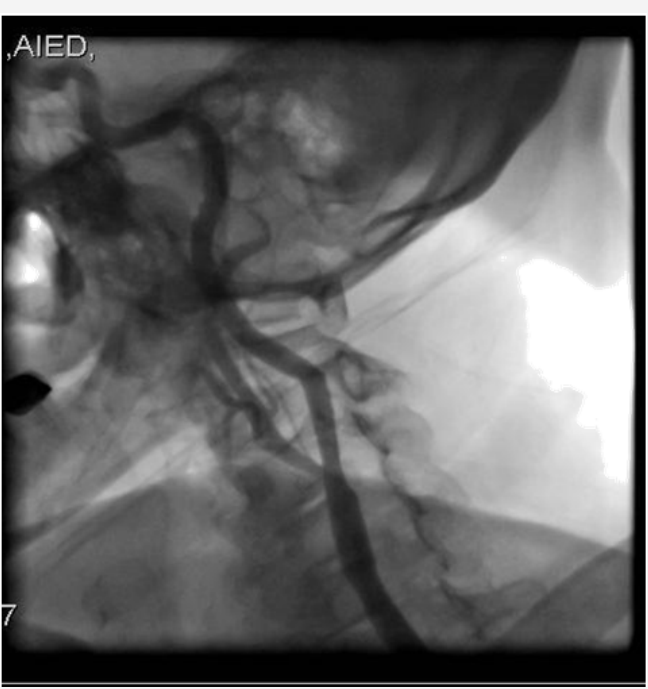

Figure 3: Post carotid stenting using embossed NAV 6 distal protection filter and $8 \times 6 \times 40 \mathrm{~mm}$ XACT self-expandable stent with excellent result.

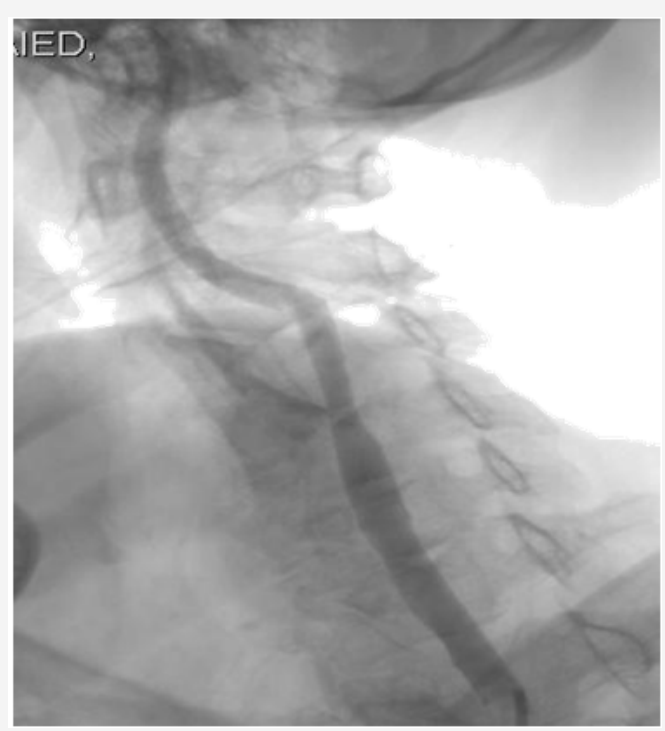

Figure 4: Repeat amigo showed wide patent stent and distal flow.
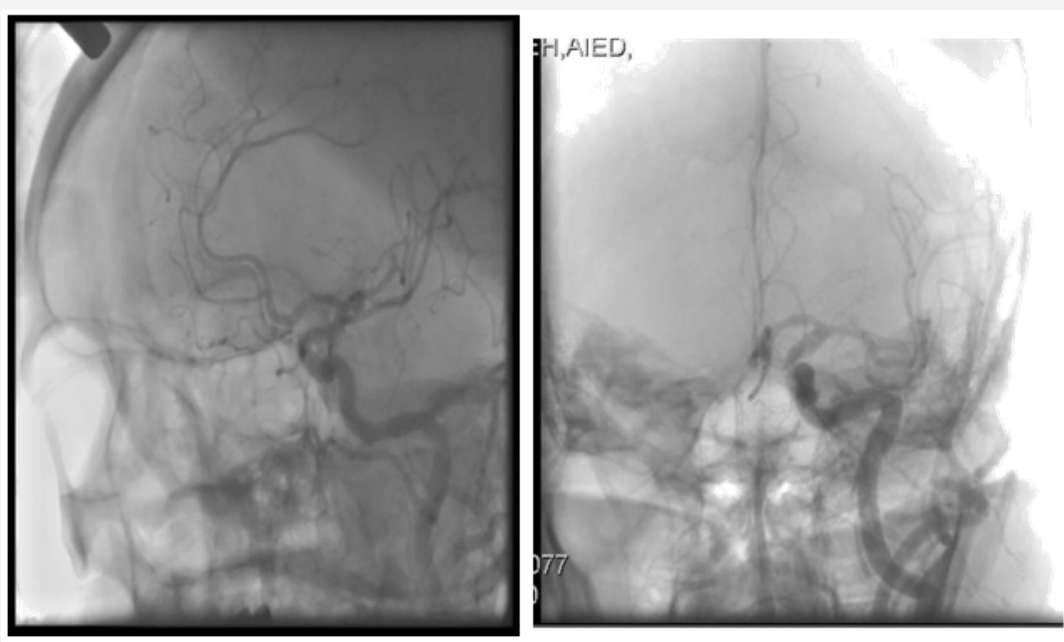

Figure 5: Cerebral angiogram revealed wide patent left anterior and middle cerebral and all branches.
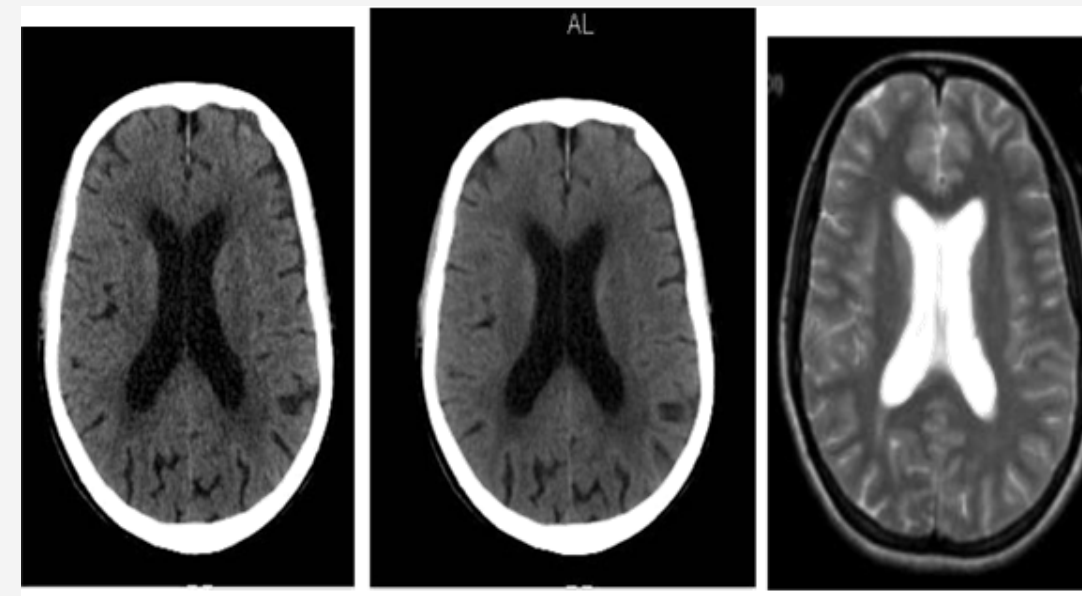

Figure 6: Unremarkable post procedure non-contrast Brain CT and MRI.

\section{Discussion}

Carotid artery angiography and stenting are becoming part of the daily intervention practice and widely performed by several subspecialties that possess the appropriate training a $\quad$ n $\quad d$ qualification. Several potential procedural and peri-procedural complications and brain injury might incur during carotid artery 
stenting (CAS), such as; major complications; embolic stroke, intracranial hemorrhage, hemodynamic events, hyper perfusion syndrome, carotid perforation, acute stent thrombosis and vascular access complications. Minor complications include: Sustained hypotension / bradycardia, Carotid artery spasm, Carotid artery dissection, Minor embolic neurological events (TIAs), and very rare Contrast induced encephalopathy (CIE). [1-9]

Rapid recognition, differential diagnosis is fundamentally important for decision making and fast management.

Our report focusses on CIE as a rare and less known complication $(0.1-1 \%)$ [10] of carotid stenting that deserve attention of the operator and all involved team to avoid unnecessary thrombolytic, invasive procedure and subsequent complications.

Contrast induced encephalopathy is the reversible neurotoxicity caused by contrast agents used during percutaneous carotid [11] and coronary interventions [12] and is defined as a transient neurological syndrome during or after the procedure mostly related to a prolonged procedure in which a large volume of contrast medium is used. The patient can develop minor or profound neurological deficits related to the involved hemisphere. Symptoms can include; motor and sensory disturbances, aphasia, vision disturbance, seizures, and transient cortical blindness (the most commonly reported symptom), with subtle or marked contrast enhancement "staining" in the basal ganglion and the cortex. Usually no angiographic vascular abnormalities are detected by intracranial angiography.

Brain computed tomography (CT) can be normal or reveals diffuse cortical and subcortical enhancement similar to subarachnoid hemorrhage (SAH) [13]. Magnetic resonance imaging (MRI) can be normal or show local or diffuse brain alteration on diffusion-weighted magnetic resonance imaging (DW-MRI).

Contrast encephalopathy can be explained as follow; given that usually contrast medium does not pass through the blood-brain barrier, this CIE phenomenon may be caused by fine particulate embolization, transient distal microcirculation spasm caused by excessive local contrast injection or in some cases contrast might cause disruption of the blood brain barrier and direct neuronal injury. Contrast-induced transient vasoconstriction has also been implicated [14-15].

Patients typically recover completely within 24 hours without a permanent neurological deficit.

From a clinical stand point, the interventionist must differentiate this benign phenomenon from a cerebral infarction, hemorrhage or hyper perfusion syndrome to avoid unnecessary drastic measures.

\section{Conclusion}

This rare phenomenon of contrast induced encephalopathy CIE as a transient neurotoxicity after carotid interventions is real (fact not fiction), must be considered and differentiated from other minor and major brain injury complications such massive cerebral infarction, bleed and hyper perfusion syndrome to avoid unnecessary aggressive intervention that may add to the stress and financial burden. The prognosis of CIE is excellent; however, subsequent contrast studies should be undertaken with extreme caution.

\section{Acknowledgement}

None.

\section{Conflict of Interest}

No Conflict of Interest.

\section{References}

1. Yadav JS, Roubin GS, Iyer S, Vitek J, King P, et al. (1997) Elective stenting of the extracranial carotid arteries. Circulation 95(2): 376-381.

2. Roubin GS, New G, Iyer SS, Vitek JJ, Al-Mubarak N, et al. (2001) Immediate and late clinical outcomes of carotid artery stenting in patients with symptomatic and asymptomatic carotid artery stenosis: a 5-year prospective analysis. Circulation 103(4): 532-537.

3. Morey SS (1998) AHA updates guidelines for carotid endarterectomy. Am Fam Physician 58(8): 1903-1904.

4. Wholey MH, Al-Mubarek N, Wholey MH (2003) Updated review of the global carotid artery stent registry. Catheter Cardiovasc Interv 60(2): 259-266.

5. Al-Mubarak N, Liu MW, Dean LS (1999) Incidences and outcomes of prolonged hypotension following carotid artery stenting. J Am Coll Cardiology 33: 65

6. Yadav JS, Wholey MH, Kuntz RE, Fayad P, Katzen BT, et al. (2004) Protected carotid-artery stenting versus endarterectomy in high-risk patients. N Engl J Med 351(15): 1493-1501.

7. Mas JL, Chatellier G, Beyssen B, Branchereau A, Moulin T, et al. (2006) Endarterectomy versus stenting in patients with symptomatic severe carotid stenosis. N Engl J Med 355(16): 1660-1671.

8. Ringleb PA, Allenberg J, Bruckmann $\mathrm{H}$, Eckstein $\mathrm{HH}$, Fraedrich $\mathrm{G}$, et al. (2006) 30-day results from the SPACE trial of stent-protected angioplasty versus carotid endarterectomy in symptomatic patients: a randomised non-inferiority trial. Lancet 368(9543): 1239-1247.

9. Cremonesi A, Setacci C, Manetti R, de Donato G, Setacci F, et al. (2005) Carotid angioplasty and stenting: lesion related treatment strategies. EuroIntervention 1(3): 289-295.

10. Menna D, Capoccia L, Rizzo AR, Sbarigia E, Speziale F (2013) An atypical case of contrast-induced encephalopathy after carotid artery stenting. Vascular 21(2): 109-112.

11. Dangas G, Monsein LH, Laureno R, Peterson MA, Laird JR, et al. (2001) Transient Contrast Encephalopathy after Carotid Artery Stenting. J Endovasc Ther 8(2): 111-113.

12. Spina R, Simon N, Markus R, Muller DW, Kathir K (2017) Contrastinduced encephalopathy following cardiac catheterization: ContrastInduced Encephalopathy. Catheter Cardiovasc Interv 90(2): 257-268.

13. Fang HY, Kuo YL, Wu CJ (2009) Transient contrast encephalopathy after carotid artery stenting mimicking diffuse subarachnoid hemorrhage: a case report. Catheter Cardiovasc Interv 73(1): 123-126.

14. Torvik A, Walday P (1995) Neurotoxicity of water-soluble contrast media. Acta Radiol Suppl 399: 221-229.

15. Caille JM, Allard M (1988) Neurotoxicity of hydrosoluble iodine contrast media. Invest Radiol 23 Suppl 1: 210-212. 